\title{
INVENTÁRIO FLORÍSTICO DE FLORESTA OMBRÓFILA DENSA DE TERRA FIRME, NA REGIÃO DO RIO URUCU- AMAZONAS, BRASIL.
}

\author{
Diógenes de Andrade de LIMA FILHO ${ }^{2}$, Francisca Dionizia de Almeida \\ MATOS ${ }^{2}$, Iêda L. do AMARAL ${ }^{2}$, Juan REVILLA ${ }^{2}$, Luiz de Souza \\ COÊLHO $^{2}$, José Ferreira RAMOS ${ }^{2}$, José Lima dos SANTOS ${ }^{2}$.
}

Resumo - O presente trabalho teve por objetivo principal caracterizar a vegetação da área de exploração de petróleo da PETROBRÁS, no rio Urucu, bem como dar subsídios para a utilização da floresta de forma organizada e produtiva, baseada em conhecimentos científicos, de modo não somente a produzir resultados econômicos mas principalmente conservar o ambiente. Os três hectares de floresta inventariada sustentam 2.241 indivíduos, abrangendo árvores, palmeiras e cipós com DAP $\geq 10 \mathrm{~cm}$, distribuídos em 577 espécies, 225 gêneros e 60 famílias. Três medidas de importância ecológica - abundância, dominância e freqüência - expressas como três porcentagens, foram somadas para obter um Índice de Valor de Importância (IVI). As duas espécies com os maiores IVIE, em toda a área pesquisada, foram Eschweilera coriacea (DC.) S. A. Mori, com 15\% no hectare 2 e E. wachenheimii (Benoist) Sandwith, com 14\% no hectare 3. As familias que obtiveram os maiores Índices de Valor de Importância (IVIF), em média, nos 3 hectares, foram Lecythidaceae $(51,6 \%)$, Sapotaceae $(40,2 \%)$ e Chrysobalanaceae $(24,6 \%)$.

Palavras-chaves: Inventário florístico, Composição Florística, Diversidade, Floresta Tropical, Amazônia,

Floristic Inventory of Three Hectares of "Terra firme" Forest in the Petroleum Drilling Region of the Urucu River in the Amazon State, Brazil.

\begin{abstract}
The aim of this paper is to characterize forest vegetation in the Urucu River region, a petroleum drilling area of PETROBRAS. This information may contribute to the organized and productive use of the forest, based on scientific knowledge, so that economic returns can be obtained while conserving the environment. All tree, vine and palm stems over $10 \mathrm{~cm} \mathrm{DBH}$ were inventoried in three hectares. A total of 2241 individuals were found in 60 families, 225 genera and 577 species or morphospecies. Three measures of ecological importance-abundance, dominance and frequency - expressed as three separate percentages were summed to give an Importance Value Index (IVIE). The two highest IVI were for Eschweilera coracea (DC.) S. A. Mori, with $15 \%$ in hectare 2, and E. wachenheimii (Benoist) Sandwith, with $14 \%$ in hectare 3 . The highest Family Importance Indices averaged across the three hectares were for Lecythidaceae (51.62\%), Sapotaceae (40.24\%) and Chrysobalanacaeae (24.56\%).
\end{abstract}

Key-words: Floristic inventory, floristic composition, diversity ,Tropical rain forest, Amazon

\section{INTRODUÇÃO}

No cenário atual, a floresta amazônica brasileira apresenta-se como tema de amplas discussões tanto no âmbito científico/ecológico, no desenvolvimento de pesquisas, quanto na sociedade de modo geral.

O conhecimento detalhado da estrutura das florestas tropicais é dificultado pela extensão da área e das várias interações entre os fatores

\footnotetext{
'Parte da dissertação de Mestrado de Diógenes de Andrade Lima Filho, financiamento INPA/ PETROBRÁS.

${ }^{2}$ Instituto Nacional de Pesquisas da Amazônia, INPA/CPBO. Av. André Araújo, 2936, Petrópolis, CEP. 69083-000, Manaus, AM. Brasil, e-mail: diogenes@inpa.gov.br
} 
ambientais bióticos e abióticos, que influenciam sobremaneira em sua composição florística.

O presente trabalho teve como objetivo principal caracterizar a vegetação da área de exploração de petróleo da PETROBRÁS, no rio Urucu, bem como dar subsídios para a utilização da floresta de forma organizada e produtiva, baseada em conhecimentos científicos, de modo não somente a produzir resultados econômicos mas principalmente conservar o ambiente.

\section{MATERIAL E MÉTODOS}

\section{Localização}

A área sob estudo está situada na Bacia do Rio Urucu, afluente da margem direita do rio Solimões, no município de Coari, Estado do Amazonas, em área de exploração de petróleo da empresa PETROBRÁS S/ $A$, entre os paralelos $4^{\circ}$ e $5^{\circ} \mathrm{S}$ e os meridianos $63^{\circ}$ e $66^{\circ}$ WGR com superfície aproximada de 514.000

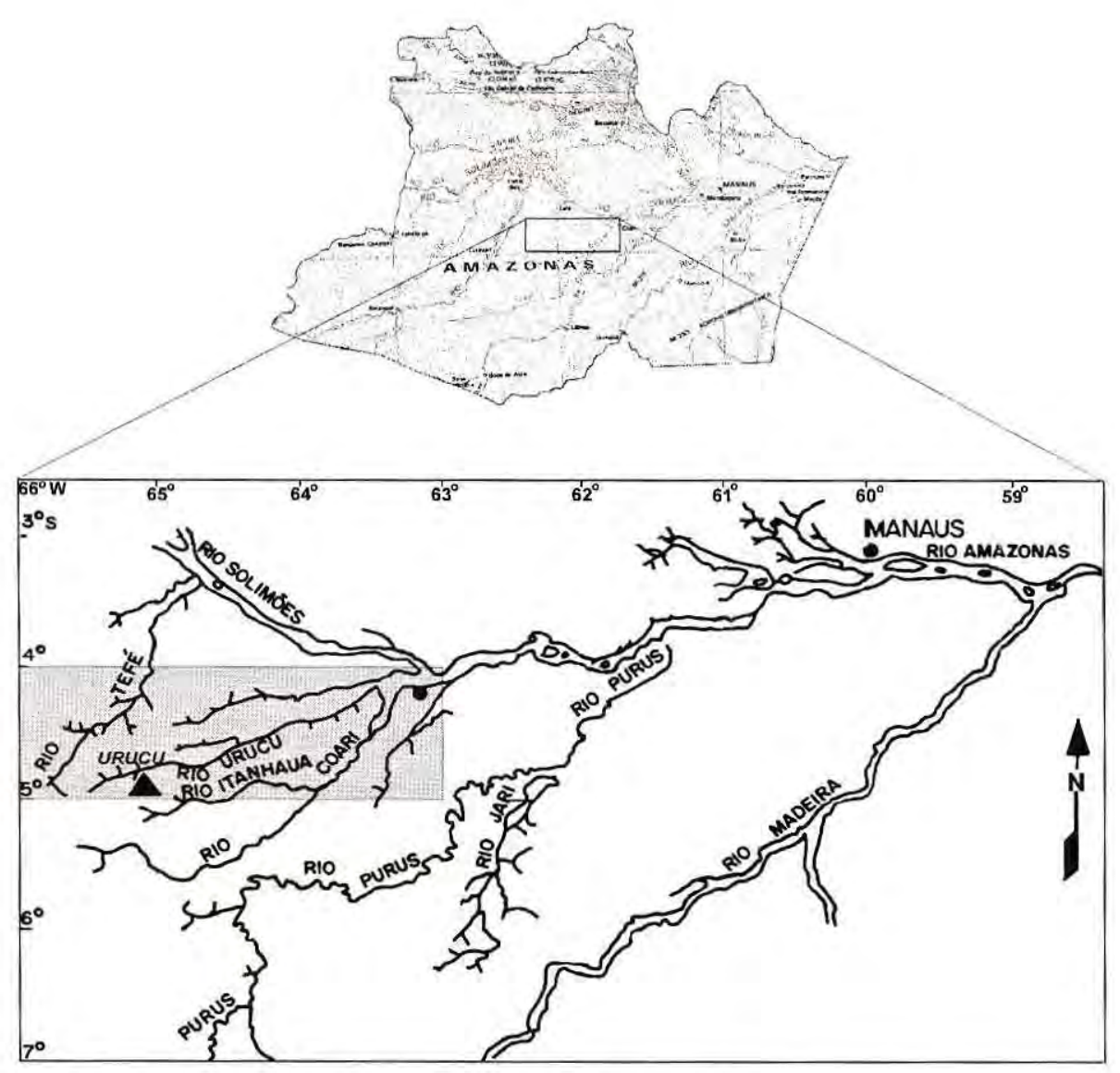

Figura 1. Localização da área de estudo, no Rio Urucu-AM. 
hectares (Fig. 1).

\section{Clima}

Segundo a classificação de Köppen (BRASIL, 1978), a região em estudo é do tipo Afi, que tem como característica principal uma flutuação anual, sendo a temperatura média mínima de $22,5^{\circ}$ $\mathrm{C}$ e a média máxima de $31,5^{\circ} \mathrm{C}$. A amplitude anual da temperatura média não ultrapassa a $5^{\circ} \mathrm{C}$. Durante o ano de 1989, a PETROBRÁS observou que a menor insolação ocorreu em março $(117,8 \mathrm{~h})$ e a maior em agosto $(203,5 \mathrm{~h})$ e a umidade relativa do ar situou-se entre 83 a $88 \%$.

Cruz (1994) citando os dados pluviométricos coletados de fevereiro/93 a janeiro/94, pela PETROBRAS, observou que o período mais chuvoso vai de setembro a abril (sendo março o mês de maior pico com $542,5 \mathrm{~mm}$ ), e o menos chuvoso de maio a agosto (tendo sido agosto o de menor pico com $80 \mathrm{~mm}$ ), e o total acumulado no período foi de $3.404 \mathrm{~mm}$.

\section{Solo}

O solo dessa região é constituido por sedimentos da "Formação Solimões", apresentando relevo ondulado, textura argilosa, bem drenado, sob floresta tropical densa (BRASIL, 1978). O mesmo é classificado como cambissolo álico, de textura média, argiloso e de consistência dura, ocorrendo em relevos que variam de suave ondulado a ondulado.

\section{Vegetação}

A região é coberta por floresta ombrófila densa de terra firme (Veloso et al., 1991), de dossel uniforme, mudando fisionomicamente em algumas áreas onde o solo é mal drenado ou existem clareiras naturais pela morte das grandes árvores.

O dossel é constituído de árvores altas que variam, em geral, de 23-32 $\mathrm{m}$, predominantemente finas $(10-20$ $\mathrm{cm}$ de DAP), troncos retilíneos, copas amplas de forma globosa, apresentando uma baixa diversidade de epifitas e lianas. A estrutura da floresta apresenta árvores que alcançam até $35 \mathrm{~m}$ de altura, evidenciando de maneira clara a existência de árvores emergentes.

\section{Metodologia}

As amostras foram alocadas, mediante a análise do mosaico fotográfico da imagem do satélite Landsat-5, de outubro de 1988, obtido no laboratório do CENPES/ PETROBRÁS através do convênio MCT/INPA/PETROBRÁS . Estas amostras foram denominadas Aeroporto (hectare 1), caracterizado por um relevo, na maior parte, plano, com presença de alguns baixios; RUC-25 (hectare 2), cujo relevo é bastante movimentado, com inclinações superior a $4^{\circ}$; e Estação de Fluidos (hectare 3), que apresentou-se mais plano em todo o transecto, não havendo variações no relevo.

A forma e o tamanho das unidades amostrais foram estabelecidas tendo por base o modelo 
proposto por Lamprecht (1964), Finol (1971); utilizado por Longhi (1980), Revilla et al. (1981), o qual consiste em uma faixa de $10 \times 1000 \mathrm{~m}$ (1 ha), dividida em parcelas de $10 \times 50 \mathrm{~m}$.

O sistema de amostragem foi $o$ sistemático-aleatório, cuja distribuição das amostras obedeceu, sistematicamente, o sentido norte-sul e alocando-as aleatoriamente nos diferentes tipos de vegetação.

A obtenção de dados no campo obedeceu ao critério de inclusão de $\mathrm{DAP}^{3} 10 \mathrm{~cm}$, os quais foram marcados com placas de alumínio e obtidas as medidas de diâmetro à altura do peito (DAP) e as alturas do fuste e total. O material botânico, fértil ou não, foi coletado, submetido à secagem e posterior identificação no herbário do INPA, cuja classificação obedece ao sistema de Cronquist (1981).

A composição florística foi analisada com base nos números de individuos, espécies, gêneros e famílias, Quociente de Mistura de Jentsch e do Grau de Homogeneidade. Segundo Hosokawa (1981), o quociente de mistura de Jentsch dá uma idéia geral da composição floristica da área em estudo. Tendo sido usado por Vega (1966, 1968) como fator de verificação da intensidade de mistura das espécies. Ainda neste sentido, foi aplicado o indice fitossociológico, Grau de Homogeneidade, o qual exprime a homogeneidade de uma associação vegetal, tendo sido relatado por Labouriau e Matos Filho (1948), Longhi (1980), Conceição (1990) e Lima Filho (1996), os quais aplicaram a equação:

$\mathrm{H}=[(\Sigma \mathrm{x}-\Sigma \mathrm{y}) / \Sigma \mathrm{N}] \mathrm{xn} \quad$ onde:

$\mathrm{H}=$ grau de homogeneidade

$\Sigma \mathrm{x}=$ número de espécies com 80 $-100 \%$ de freqüência absoluta (ocorrência nas parcelas amostrais)

$\Sigma y=$ número de espécies com 0 $20 \%$ de freqüência absoluta (ocorrência nas parcelas amostrais)

$\Sigma \mathrm{N}=$ número total de espécies

$\mathrm{n}=$ número de classes de freqüência

A estrutura horizontal foi analisada através de critérios que indicam a ocupação do solo pela espécie, no sentido horizontal, utilizando-se os parâmetros de abundância absoluta (Lamprecht, 1962; 1964), calculada pela contagem do número total de individuos de uma mesma espécie, em relação à unidade de área e a abundância relativa, foi definida pela percentagem da abundância absoluta. A dominância absoluta (Cain et al., 1956; Lamprecht, 1964; Finol, 1971; Hosokawa, 1981), obtida pela soma das áreas das secções transversais dos indivíduos de uma espécie por unidade de área, enquanto que a dominância relativa é calculada pela percentagem do total das dominâncias absolutas (área basal/ha), e corresponde à participação em percentagem de cada espécie na expansão horizontal total. A freqüência absoluta (Förster, 1973) representa o número de subparcelas em que ocorre uma espécie, dividido pelo número total de subparcelas em que foi dividida a amostra e a relativa é em relação à soma das frequuências 
absolutas das parcelas. O indice de valor de importância específica (IVIE) segundo Curtis \& McIntosch (1950), utilizado por Cain et al. $(1956 ; 1959)$, Matos \& Amaral (1999), representa o somatório dos valores relativos de abundância, dominância e freqüência de cada espécie e o indice de Valor de importância familiar (IVIF) proposto por Mori et al. (1983), obtêm-se através do somatório da diversidade, densidade e dominância relativa das familias.

\section{RESULTADOS E DISCUSSÃO}

Nos três hectares inventariados, foram registrados 2.241 indivíduos com DAP $\geq 10 \mathrm{~cm}$, distribuídos em 60 familias, 225 gêneros e 577 espécies, incluindo árvores, cipós e palmeiras. O sistema adotado para a classificação das familias foi o de Cronquist (1981).

A área mais representativa foi o Ruc-25 com 769 individuos, classificados em 48 famílias, 155 gêneros e 322 espécies. A menos representativa foi o hectare1, próximo ao aeroporto, tanto em número de individuos, quanto em familias e espécies, tendo obtido 710 individuos, classificados em 44 familias, 126 gêneros e 254 espécies.

A Tabela 1 mostra a distribuição geral do número de indivíduos, gêneros e espécies por familia botânica com DAP $\geq 10 \mathrm{~cm}$, considerando a área total de $30.000 \mathrm{~m}^{2}$.

As famílias Lecythidaceae, Sapotaceae, Chrysobalanaceae, Myristicaceae e Moraceae foram as mais representativas, com referência ao número de indivíduos, obtendo 501; 284; 191; 132 e 117, respectivamente. Estas 5 famílias representam conjuntamente $54,66 \%$ de todos os indivíduos encontrados. Por outro lado, as familias Araliaceae, Cochlospermaceae, Gnetaceae, Lacistemaceae, Ochnaceae, Polygonaceae, Simaroubaceae, Strelitziaceae e Verbenaceae, obtiveram apenas 1 individuo, que somados representam $0,40 \%$ do total dos individuos registrados.

Observe-se, ainda na Tabela 1, que as familias Sapotaceae, Euphorbiaceae, Annonaceae, Caesalpiniaceae, Fabaceae, Mimosaceae e Rubiaceae foram as que obtiveram maior número de gênero com $21 ; 15 ; 11 ; 10 ; 10 ; 10$ e 10 , respectivamente; representando $38,66 \%$ do total, enquanto $33,33 \%$ das familias estudadas apresentaram somente 1 gênero.

Em relação ao número de espécies, as famílias mais diversificadas foram Sapotaceae, com 68; Chrysobalanaceae, com 50; Lecythidaceae e Mimosaceae, com 35; Burseraceae, com 32; e Caesalpiniaceae, com 31, obtendo assim uma percentagem de $43,50 \%$ do total global das espécies inventariadas. Em contraparte, $25 \%$ das famílias encontradas apresentaram somente 1 espécie; não que elas sejam monoespecificas, mas porque neste local, a maioria, são famílias constituídas de poucas espécies, portanto, mais difíceis de serem encontradas.

Os resultados desta pesquisa estão bem próximos aqueles encontrados por Jardim (1985), na área da "Bacia 3", do projeto "Manejo Ecológico e Exploração da Floresta ] 
Tabela 1. Distribuição do número de individuos (DAP $\geq 10 \mathrm{~cm}$ ), gêneros e espécies por familia nos 3 hectares de floresta ombrófila densa de terra firme na área do Rio Urucu - AM.

\begin{tabular}{|c|c|c|c|c|c|c|c|c|c|c|}
\hline \multirow[t]{2}{*}{$N^{0}$} & \multirow[t]{2}{*}{ Familia } & \multicolumn{3}{|l|}{ Hectare 1} & \multicolumn{3}{|c|}{ Hectare 2} & \multicolumn{3}{|l|}{ Hectare 3} \\
\hline & & individ, & gên. & espéc. & indiv. & gên. & espéc. & individ. & gên. & espéc. \\
\hline 1 & Anacardiaceae & 4 & 2 & $\cdot 4$ & 3 & 2 & 3 & 6 & 3 & 3 \\
\hline 2 & Annonaceae & 8 & 3 & 3 & 16 & 8 & 12 & 18 & 7 & 11 \\
\hline 3 & Apocynaceae & 9 & 6 & 6 & 7 & 5 & 6 & 7 & 3 & 4 \\
\hline 4 & Araliaceae & & & & 1 & 1 & 1 & & & \\
\hline 5 & Arecaceae & 23 & 4 & 6 & 12 & 4 & 6 & 35 & 4 & 6 \\
\hline 6 & Bignoniaceae & & & & 2 & 1 & 1 & 1 & 1 & 1 \\
\hline 7 & Bixaceae & & & & 1 & 1 & 1 & & & \\
\hline 8 & Bombacaceae & 7 & 4 & 5 & 15 & 4 & 4 & 6 & 3 & 4 \\
\hline 9 & Boraginaceae & 1 & 1 & 1 & 1 & 1 & 1 & & & \\
\hline 10 & Burseraceae & 40 & 3 & 16 & 20 & 3 & 10 & 40 & 5 & 23 \\
\hline 11 & Caesalpiniaceae & 28 & 8 & 16 & 55 & 7 & 20 & 31 & 6 & 14 \\
\hline 12 & Caryocaraceae & 3 & 2 & 3 & 3 & 1 & 1 & 4 & 1 & 1 \\
\hline 13 & Celastraceae & 1 & 1 & 1 & 1 & 1 & 1 & & & \\
\hline 14 & Cecropiaceae & 4 & 3 & 4 & 12 & 2 & 6 & 6 & 1 & 3 \\
\hline 15 & Chrysobalanaceae & 47 & 3 & 23 & 78 & 5 & 33 & 66 & 4 & 19 \\
\hline 16 & Clusiaceae & 13 & 3 & 4 & 12 & 4 & 7 & 7 & 4 & 6 \\
\hline 17 & Combretaceae & & & & 2 & 2 & 2 & 7 & 1 & 4 \\
\hline 18 & Dichapetalaceae & 1 & 1 & 1 & 1 & 1 & 1 & & & \\
\hline 19 & Dilleniaceae & & & & 2 & 1 & 1 & & & \\
\hline 20 & Elaeocarpaceae & 12 & 1 & 4 & 10 & 1 & 6 & 8 & 1 & 7 \\
\hline 21 & Euphorbiaceae & 37 & 10 & 13 & 53 & 14 & 20 & 10 & 4 & 5 \\
\hline 22 & Fabaceae & 9 & 6 & 8 & 11 & 7 & 8 & 12 & 4 & 8 \\
\hline 23 & Flacourtiaceae & & & & 2 & 1 & 2 & 2 & 1 & 1 \\
\hline 24 & Gnetaceae & & & & 1 & 1 & 1 & & & \\
\hline 25 & Hippocrateaceae & & & & & & & 2 & 1 & 2 \\
\hline 26 & Humiriaceae & 2 & 2 & 2 & 9 & 2 & 3 & 7 & 3 & 4 \\
\hline 27 & Icacinaceae & 1 & 1 & 1 & & & & 1 & 1 & 1 \\
\hline 28 & Lacistemaceae & 1 & 1 & 1 & & & & & & \\
\hline 29 & Lauraceae & 40 & 5 & 11 & 21 & 5 & 10 & 17 & 4 & 8 \\
\hline 30 & Lecythidaceae & 154 & 5 & 19 & 161 & 5 & 23 & 186 & 4 & 20 \\
\hline
\end{tabular}


Tabela 1. Continuação

\begin{tabular}{|c|c|c|c|c|c|c|c|c|c|c|}
\hline \multirow[t]{2}{*}{$\mathrm{N}^{2}$} & \multirow[t]{2}{*}{ Familla } & \multicolumn{3}{|c|}{ Hectare 1} & \multicolumn{3}{|c|}{ Hectare 2} & \multicolumn{3}{|c|}{ Hectare 3} \\
\hline & & individ. & gên. & espéc. & indiv. & gên. & espéc. & individ. & gên. & espéc, \\
\hline 31 & Loganiaceae & 3 & 1 & 1 & & & & & & \\
\hline 32 & Malpighiaceae & & & & 4 & 2 & 4 & & & \\
\hline 33 & Melastomataceae & 2 & 1 & 2 & 5 & 4 & 5 & 1 & 1 & 1 \\
\hline 34 & Meliaceae & 2 & 1 & 2 & 4 & 1 & 3 & 1 & 1 & 1 \\
\hline 35 & Meliosmaceae & & & & 1 & 1 & 1 & 1 & 1 & 1 \\
\hline 36 & Menispermaceae & 2 & 2 & 2 & 1 & 1 & 1 & & & \\
\hline 37 & Mimosaceae & 30 & 6 & 14 & 22 & 5 & 17 & 35 & 8 & 19 \\
\hline 38 & Monimiaceae & 1 & 1 & 1 & 4 & 1 & 1 & 2 & 1 & 2 \\
\hline 39 & Moraceae & 38 & 6 & 15 & 35 & 6 & 16 & 44 & 6 & 13 \\
\hline 40 & Myristicaceae & 54 & 4 & 14 & 33 & 2 & 16 & 45 & 3 & 17 \\
\hline 41 & Myrsinaceae & 2 & 2 & 2 & & & & & & \\
\hline 42 & Myrtaceae & 3 & 2 & 2 & 12 & 3 & 5 & 2 & 2 & 2 \\
\hline 43 & Nyctaginaceae & 7 & 1 & 1 & 7 & 1 & 2 & 11 & 1 & 3 \\
\hline 44 & Ochnaceae & & & & & & & 1 & 1 & 1 \\
\hline 45 & Olacaceae & 7 & 2 & 4 & 15 & 4 & 6 & 15 & 2 & 6 \\
\hline 46 & Polygalaceae & 2 & 1 & 1 & & & & & & \\
\hline 47 & Polygonaceae & & & & & & & 1 & 1 & 1 \\
\hline 48 & Quiinaceae & & & & 2 & 2 & 2 & & & \\
\hline 49 & Rhizophoraceae & 1 & 1 & 1 & 1 & 1 & 1 & 2 & 1 & 1 \\
\hline 50 & Rosaceae & 2 & 1 & 1 & & & & & & \\
\hline 51 & Rubiaceae & 3 & 2 & 2 & 10 & 7 & 7 & 7 & 4 & 4 \\
\hline 52 & Sapindaceae & 1 & 1 & 1 & 3 & 3 & 3 & 3 & 2 & 2 \\
\hline 53 & Sapotaceae & 98 & 10 & 29 & 88 & 16 & 36 & 98 & 12 & 31 \\
\hline 54 & Simaroubaceae & 1 & 1 & 1 & & & & & & \\
\hline 55 & Sterculiaceae & 3 & 2 & 3 & 5 & 2 & 3 & 6 & 2 & 2 \\
\hline 56 & Strelitziaceae & & & & & & & 1 & 1 & 1 \\
\hline 57 & Tiliaceae & 1 & 1 & 1 & 1 & 1 & 1 & 3 & 2 & 2 \\
\hline 58 & Verbenaceae & & & & & & & 1 & 1 & 1 \\
\hline 59 & Violaceae & & & & 3 & 1 & 1 & 1 & 1 & 1 \\
\hline \multirow[t]{2}{*}{60} & Vochysiaceae & 2 & 2 & 2 & 1 & 1 & 1 & 2 & 2 & 2 \\
\hline & TOTAL & 710 & 126 & 254 & 769 & 155 & 322 & 762 & 122 & 269 \\
\hline
\end{tabular}


As 30 espécies mais abundantes nos 3 hectares representam em média $42,66 \%$ do total da floresta estudada. Mas, quando se analisa cada hectare têm-se $45,04 \%$ para o hectare 1 ; $35,75 \%$ para o hectare 2 ; e $47,21 \%$ para o hectare 3 (Tabs 4,5 e 6 ), demonstrando que o número de indivíduos por espécie, varia de local para local e que esta variação é da ordem de 6 pontos percentuais para este estudo.

As 30 espécies que apresentaram maior dominância representam em média $50,46 \%$ da área basal total. Entretanto, quando se analisa cada hectare tem-se $51,45 \%$ no hectare 1 ; $47,37 \%$ no hectare 2 ; e $52,55 \%$ no hectare 3 (Tabs 4, 5 e 6 ).

As espécies que apresentaram maiores valores de dominância nos 3 hectares foram Dinizia excelsa, com $2,79 \mathrm{~m}^{2}$ no hectare 2 , e Buchenavia parvifolia, com $1,80 \mathrm{~m}^{2}$ no hectare 3 . Estas espécies, embora não tenham apresentado um alto valor de abundância e freqüência na área, são consideradas dominantes, justificandose pelo seu porte volumoso. Esses resultados confirmam o postulado por Finol (1971), quando diz que quase sempre as espécies que representam a potencialidade da floresta, ou que determinam a qualidade de sítio em uma área, estão classificadas entre as primeiras nos parâmetros estruturais avaliados.

Analisando as 30 espécies mais freqüentes na área tem-se uma média de $33,81 \%$. Mas, quando se calcula para cada hectare, essa percentagem é de $38,51 \%$ para o hectare $1 ; 27,03 \%$ para o hectare 2; e $35,88 \%$ para o hectare 3 (Tabs 4, 5 e 6 ). As espécies mais freqüentes foram $E$. coriacea, com $2,79 \%$ no hectare 2 ; e $2,74 \%$ no hectare 1, seguida da E. wachenheimii, com $2,58 \%$ no hectare 3 .

As 30 espécies com maiores valores de Índice de Valor de Importância Especifica (IVIE), nas 3 áreas apresentam uma média de 126,95 (IVIE) representando $42,32 \%$ do total. Mas, individualmente, no hectare 1 , com um total de 135,01 (IVIE) representam 45\%; no hectare $2 \mathrm{com}$ 110,16 (IVIE) representam $36,72 \%$; e no hectare 3 com 135,69 (IVIE) representam $45,23 \%$ do índice de valor de importância (Tabs 4, 5 e 6).

Dentre as 30 , as espécies que obtiveram os maiores índices de valor de importância nos 3 hectares estudados foram $E$. coriacea, com $15 \%$ no hectare 2 , seguida da $E$. wachenheimii, com $14 \%$ no

Tabela 2. Quociente de mistura de Jentsch na área de estudo do Rio Urucu-AM.

\begin{tabular}{cccc}
\hline Ha. & Spp. & Ind. & QM. \\
\hline 1 & 253 & 710 & $1: 3$ \\
2 & 322 & 769 & $1: 2$ \\
3 & 269 & 762 & $1: 3$ \\
Média & 281,33 & 747 & $1: 2,6$ \\
\hline Total & 844 & 2.241 & \\
\hline
\end{tabular}

$\mathrm{Ha}=$ hectare; $\mathrm{Spp}=$ No. de espécies; Ind.=No. de árvores e cipós $(\mathrm{DAP} \geq 10 \mathrm{~cm})$; $\mathrm{QM}=$ Quociente de Mistura de Jentsch 
Tabela 3. Grau de homogeneidade floristica das 3 áreas estudas do Rio Urucu-AM.

\begin{tabular}{ccccc}
\hline Amostra & $\mathrm{X}$ & $\mathrm{Y}$ & $\mathrm{N}$ & $\mathrm{H}$ \\
\hline 1 & 1 & 220 & 253 & $-4,33$ \\
2 & 1 & 300 & 322 & $-4,64$ \\
3 & 0 & 245 & 269 & $-4,55$ \\
Média & 0,67 & 255 & 281,33 & $-4,51$ \\
\hline
\end{tabular}

$X=n^{\circ}$ de espécies com $80-100 \%$ de freqüência absoluta; $Y=n^{\circ}$ de espécies com 0 $20 \%$ de freqüência absoluta

$\mathrm{N}=\mathrm{n}^{\circ}$ total de espécies; $\mathrm{H}=\mathrm{Grau}$ de homogeneidade

hectare 3 .

A família Lecythidaceae, nos três hectares inventariados, obteve a melhor classificação no Índice de Importância Familiar (IVIF), apresentando 51,$53 ; 52,19$ e $51,13 \%$ respectivamente, com média de $51,62 \%$; seguida da familia Sapotaceae com 45,$23 ; 36,80 ; 38,68 \%$ nos hectares 1,2 e 3 e média $40,24 \%$. Em terceiro lugar está a familia Chrysobalanaceae com média de $24,46 \%$ por hectare (Tab. 7 ).

A percentagem média para as 10 principais familias, com maiores valores de IVIF, ficou em média 218,76 (IVIF), representando $72,92 \%$ do IVIF total. Quando estas familias são analisadas nos hectares individualmente tem-se 229,25 (IVIF) no hectare 1, representando $76,42 \%$; hectare $2 \mathrm{com}$ 213,99 (IVIF), representando $71,33 \% \mathrm{e}$; hectare 3 com 213,06 (IVIF), representando $71,02 \%$.

A distribuição dos indivíduos inventariados nos 3 hectares, por classe de DAP, apresentada na figura 2 , mostra que as classes inferiores, contidas no intervalo $10-25 \mathrm{~cm}$ de DAP, englobou o maior número de indivíduos, com média de 567 indivíduos/ha e representam mais de
$75 \%$ da abundância total.

O hectare 3 (estação de fluidos) apresentou o maior número de indivíduos na classe inferior (10 $25 \mathrm{~cm}$ de DAP), com 576 representando $75,59 \%$ da abundância, seguido pelo hectare 2 (RUC-25), com 573 indivíduos representando $74,51 \%$ do total deste parâmetro.

Nas classes superiores, correspondentes àquelas com DAP > $40 \mathrm{~cm}$, o número de indivíduos ficou com média em torno de 52 árvores por hectare, representando $6,54 \%$ da média das abundância totais. Mas, quando os três hectares foram analisados individualmente tem-se 46 árvores no hectare 1 representando $6,49 \%$ do total; no hectare 2 e 3 , respectivamente, obteve-se 55 individuos cada um representando $6,57 \%$.

\section{CONCLUSÃO}

Os individuos arbóreos estudados são mais finos, estruturalmente, que em outros locais da Amazônia brasileira, haja vista o alto percentual representativo nas classes inferiores, contidas no intervalo de $10-25 \mathrm{~cm}$, representando mais de $75 \%$ de todos os individuos registrados. Uma vez que a 
interferência antrópica, constatada visualmente, na área é baixa, deduz-se que o local é constituído de uma floresta jovem, em formação, com alta variação tanto genética quanto em idade, não tendo atingido, portanto, o seu estágio clímax de desenvolvimento.

A diversidade específica, demonstrada através do Quociente de Mistura de Jentsch, mostrou que a proporcionalidade de indivíduos por espécie é bem baixa, denotando a alta heterogeneidade entre as espécies que compõem a flora local.

A abundância total, encontrada na área de estudo, está representada

Tabela 4. Relação das 30 espécies com maior Índice de Valor de Importância Específica - IVIE no hectare 1 na área do Rio Urucu - AM.

\begin{tabular}{|c|c|c|c|c|c|}
\hline Ne & Espécie & $A(\%)$ & $\mathrm{D}(\%)$ & $F(\%)$ & IVIE $(\%)$ \\
\hline 1 & Eschweilera coriacea (DC.) S.A. Mori & 4,64 & 4,51 & 2,74 & 11,89 \\
\hline 2 & Chrysophyllum sp. & 2,11 & 6,00 & 1,54 & 9,65 \\
\hline 3 & Eschweilera $\mathrm{sp}$. & 2,67 & 4,56 & 2,23 & 9,46 \\
\hline 4 & Eschweilera wachenheimii (Benoist) Sandwith & 3,52 & 2,33 & 2,23 & 8,08 \\
\hline 5 & Eschweilera chartaceifolia Mori & 3,80 & 1,95 & 2,06 & 7,81 \\
\hline 6 & Eschweilera decolorans Sandw. & 1,54 & 4,15 & 1,20 & 6,89 \\
\hline 7 & Ocotea sp. & 1,54 & 3,68 & 1,20 & 6,42 \\
\hline 8 & Eschweilera collina Eyma. & 1,97 & 1,73 & 1,54 & 5,25 \\
\hline 9 & Pouteria guianensis Aubl. & 1,40 & 2,35 & 1,37 & 5,12 \\
\hline 10 & Micropholis mensalis (Baekni) Aubrév. & 1,26 & 1,53 & 1,37 & 4,16 \\
\hline 11 & Chrysophyllum sanguinolentum (Pierre) Baehni. & 1,26 & 1,76 & 1,03 & 4,05 \\
\hline 12 & Inga sp. & 1,40 & 0,88 & 1,71 & 3,99 \\
\hline 13 & Sloanea sp. & 1,26 & 1,12 & 1,54 & 3,92 \\
\hline 14 & Micropholis sp. & 1,40 & 1,28 & 1,20 & 3,88 \\
\hline 15 & Iryanthera tricornis Ducke & 1,54 & 081 & 1,37 & 3,72 \\
\hline 16 & Oenocarpus bataua Mart. & 1,69 & 0,96 & 1,03 & 3,68 \\
\hline 17 & Iryanthera laevis Markgr. & 1,12 & 1,06 & 1,20 & 3,38 \\
\hline 18 & Iryanthera ulei Ducke & 1,26 & 0,35 & 1,54 & 3,15 \\
\hline 19 & Lauraceae (Indeterminada) & 0,98 & 0,80 & 1,20 & 2,98 \\
\hline 20 & Brosimum rubescens Taub. & 0,84 & 1,22 & 0,85 & 2,91 \\
\hline 21 & Ocotea opifera Mart. & 0.84 & 1.17 & 0,85 & 2,86 \\
\hline 22 & Neea sp. & 0,98 & 0,53 & 1,20 & 2,71 \\
\hline 23 & Mabea sp. & 1,26 & 0,34 & 1,03 & 2,63 \\
\hline 24 & Monopteryx inpae W. Rodr. & 0,14 & 2,23 & 0,17 & 2,54 \\
\hline 25 & Pseudolmedia sp. & 0,70 & 0,81 & 0,85 & 2,36 \\
\hline 26 & Chrysophyllum auratum Miq. & 0,70 & 0,79 & 0,85 & 2,34 \\
\hline 27 & Helicostylis scabra (J.F. Macbr.) C.C. Berg. & 0,70 & 0,78 & 0,85 & 2,33 \\
\hline 28 & Oenocarpus bacaba Mart. & 0,98 & 0,32 & 1,03 & 2,33 \\
\hline 29 & Sclerolobium sp. & 0,84 & 0,76 & 0,68 & 2,28 \\
\hline 30 & Licania sp. & 0,70 & 0,69 & 0,85 & 2,24 \\
\hline
\end{tabular}

$(A \%)=$ Abundância relativa; $(D \%)=$ Dominância relativa; $(F \%)=$ Frequência relativa;

IVIE $(\%)=$ Indice de Valor de Importância Específica. 
Tabela 5. Relação das 30 espécies com maior Indice de Valor de Importância Especifica - IVIE (DAP $\geq 10 \mathrm{~cm}$ ) no hectare 2 na área do Rio Ururu - AM.

\begin{tabular}{|c|c|c|c|c|c|}
\hline № & Espécie & $A(\%)$ & $D(\%)$ & $F(\%)$ & $\operatorname{IVIE}(\%)$ \\
\hline 1 & Eschweilera coriacea (DC.) S.A. Mori & 8,06 & 4,15 & 2,79 & 15,00 \\
\hline 2 & Dinizia excelsa Ducke & 0,13 & 7,63 & 0,15 & 7,91 \\
\hline 3 & Eschweilera wachenhenii (Benoist) Sandw. & 2,34 & 1,79 & 1,15 & 5,68 \\
\hline 4 & Micropholis guyanensis Pierre. & 2,21 & 1,34 & 1,70 & 5,25 \\
\hline 5 & Scleronema micranthum Ducke. & 1,04 & 3,11 & 0,93 & 5,08 \\
\hline 6 & Eschweilera collina Eyma & 1,95 & 1,57 & 1,39 & 4,91 \\
\hline 7 & Licania micrantha Miq. & 1,30 & 1,51 & 1,24 & 4,05 \\
\hline 8 & Eschweilera cyathiformis S.A. Mori & 0,65 & 2,76 & 0,62 & 4,03 \\
\hline 9 & Lecythis retusa Spruce ex O. Berg. & 0,26 & 3,40 & 0,31 & 3,97 \\
\hline 10 & Pouteria sp. & 0,78 & 1,92 & 0,93 & 3,63 \\
\hline 11 & Eschweilera atropetiolata S.A. Mori & 1,04 & 1,66 & 0,77 & 3,47 \\
\hline 12 & Cariniana micrantha Ducke & 0,52 & 2,33 & 0,62 & 3,47 \\
\hline 13 & Ecclinusa guianensis Eyma & 0,78 & 1,82 & 0,46 & 3,06 \\
\hline 14 & Swartzia argentea Spruce ex Benth. & 1,30 & 0,35 & 1,39 & 3,05 \\
\hline 15 & Sagotia racemosa Baill. & 1,56 & 0,47 & 0,77 & 2,80 \\
\hline 16 & Minquartia guianensis Aubl. & 1,04 & 0,97 & 0,77 & 2,78 \\
\hline 17 & Eschweilera grandiflora (Aubl.) Sandwith & 1,17 & 0,53 & 1,08 & 2,78 \\
\hline 18 & Couratari stellata A.C. Sm. & 0,65 & 1,22 & 0,77 & 2,64 \\
\hline 19 & Dimorphandra sp. & 0,13 & 2,22 & 0,15 & 2,50 \\
\hline 20 & Sacoglottis guianensis Benth. & 0,91 & 0,70 & 0,77 & 2.38 \\
\hline 21 & Ocotea sp. & 0,91 & 0,66 & 0,77 & 2,34 \\
\hline 22 & Iryanthera ulei Ducke & 0,91 & 0,31 & 1,08 & 2,30 \\
\hline 23 & Chrysoplyllum sanguinolentum (Pierre) Baehni & 0,78 & 0,88 & 0,62 & 2,28 \\
\hline 24 & Eschweilera bracteosa (Poepp. ex O.Berg.) Miers & 0,65 & 0,98 & 0,62 & 2,25 \\
\hline 25 & Couepia obovata Ducke & 0,91 & 0,36 & 0,93 & 2,20 \\
\hline 26 & Heterostemon ellipticus Mart. ex Benth. & 0,91 & 0,16 & 1,08 & 2,15 \\
\hline 27 & Swartzia laevicarpa Amshoff & 0,65 & 0,69 & 0,77 & 2,11 \\
\hline 28 & Brosimum lactescens (S. Moore.) C.C. Berg. & 0,91 & 0,36 & 0,77 & 2,04 \\
\hline 29 & Pouteria guianensis Aubl. & 0,65 & 0,93 & 0,46 & 2,04 \\
\hline 30 & Pogonophora schumburgkiana Miers ex Benth. & 0,65 & 0,59 & 0,77 & 2,01 \\
\hline
\end{tabular}

$(A \%)=$ Abundância relativa; $(D \%)=$ Dominância relativa; $(F \%)=$ Frequência relativa IVIE $(\%)=$ Índice de Valor de Importância Específica. 
Tabela 6. Relação das 30 espécies com maior Índice de Valor de Importância Específica - IVIE $(D A P \geq 10 \mathrm{~cm})$ no hectare 3 na área do Rio Ururu - AM.

\begin{tabular}{|c|c|c|c|c|c|}
\hline № & Espécie & $A(\%)$ & $\mathrm{D}(\%)$ & $F(\%)$ & IVIE(\%) \\
\hline 1 & Eschweilera wachenhemii (Benoist) Sandwith & 7,48 & 3,93 & 2,58 & 13,99 \\
\hline 2 & Chrysophyllum sanguinolentum (Pierre) Baehni & 2,62 & 4,59 & 2,06 & 9,27 \\
\hline 3 & Eschweilera tessmannii R. Knuth. & 4,19 & 2,13 & 2,41 & 8,73 \\
\hline 4 & Eschweilera coriacea (DC.) S.A. Mori. & 3,41 & 2,36 & 2,06 & 7,83 \\
\hline 5 & Buchenavia parvifolia Ducke & 0,52 & 5,52 & 0,68 & 6,72 \\
\hline 6 & Oenocarpus bataua Mart. & 3,01 & 2,09 & 1,55 & 6,65 \\
\hline 7 & Brosimum rubescens Taub. & 1,70 & 3,27 & 1,55 & 6,52 \\
\hline 8 & Chrysophyllum sp. & 2,09 & 2,57 & 1,55 & 6,21 \\
\hline 9 & Eschweilera sp. & 1,96 & 1,94 & 1,72 & 5,62 \\
\hline 10 & Pseudolmedia laevis (Ruiz. \& Pav.) J.F. Macbr. & 1,44 & 1,46 & 1,55 & 4,45 \\
\hline 11 & Licania sp. & 1,44 & 1,61 & 1,37 & 4,42 \\
\hline 12 & Corythophora alta R. Kunth. & 2,09 & 1,13 & 1,03 & 4,25 \\
\hline 13 & Swartzia argentea Spruce. Ex Benth. & 1,31 & 1,26 & 1,55 & 4,12 \\
\hline 14 & Licania egleri Prance. & 1,44 & 1,22 & 1,37 & 4,03 \\
\hline 15 & Ocotea sp. & 1,18 & 1,82 & 1,03 & 4,03 \\
\hline 16 & Sacoglottis sp. & 0,52 & 2,78 & 0,68 & 3,98 \\
\hline 17 & Licania micrantha Miq. & 1,31 & 0,61 & 1,20 & 3,12 \\
\hline 18 & Licania hypoleuca Benth. & 1,04 & 0,84 & 0,86 & 2,74 \\
\hline 19 & Micropholis casiquiarensis Aubrév. & 0,78 & 1,07 & 0,86 & 2,71 \\
\hline 20 & Eschweilera bracteosa (Poepp. ex O.Berg.) Miers & 1,18 & 0,62 & 0,86 & 2,66 \\
\hline 21 & Micropholis sp. & 0,91 & 0,68 & 1,03 & 2,62 \\
\hline 22 & Hymenolobium excelsum Ducke & 0,39 & 1,62 & 0,51 & 2,52 \\
\hline 23 & Caryocar glabrum (Aubl.) Pers. & 0,52 & 1,27 & 0,68 & 2,47 \\
\hline 24 & Swartzia polyphylla DC. & 0,52 & 1,26 & 0,68 & 2,46 \\
\hline 25 & Iryanthera paradoxa (Schwacke) Warb. & 1,04 & 0,31 & 1,03 & 2,38 \\
\hline 26 & Sclerolobium sp. & 0,52 & 1,13 & 0,68 & 2,33 \\
\hline 27 & Buchenavia grandis Ducke & 0,13 & 2,03 & 0,17 & 2,33 \\
\hline 28 & Xylopia sp. & 0,13 & 0,63 & 0,86 & 2,32 \\
\hline 29 & Eschweilera grandiflora (Aubl.) Sandwith & 0,91 & 0,38 & 0,86 & 2,15 \\
\hline 30 & Iryanthera juruensis Warb. & 0,78 & 0,42 & 0,86 & 2,06 \\
\hline
\end{tabular}

$(A \%)=$ Abunndância relativa; $(D \%)=$ Dominância relativa; $(F \%)=$ Frequência relativa IVIE $(\%)=$ indice de Valor de Importância Específica. . 
em mais de $50 \%$, por individuos das familias Lecythidaceae, Sapotaceae, Chrysobalanaceae, Myristicaceae e Moraceae.

Quanto ao IVIE as espécies $E$. coriacea e E. wachenheimii apresentaram a distribuição mais regular em toda área, com médias de $11,58 \%$ e $9,25 \%$ do IVI respectivamente. Em relação ao IVIF, as famílias Lecythidaceae e Sapotaceae foram os destaques, com

Tabela 7. Relação das 10 familias com maior Indice de Valor de Importância Familiar - IVIF $(\mathrm{DAP} \geq 10 \mathrm{~cm})$ nos 3 hectares estudados de floresta ombrofila densa de terra firme na área do Rio Urucu - AM.

\begin{tabular}{clcccc}
\hline $\mathrm{N}^{0}$ & Especie & Den.(\%) & Div. $(\%)$ & Dom.(\%) & IVIF (\%) \\
\hline & & Hectare 1 & & & \\
1 & Lecythidaceae & 21,69 & 7,50 & 22,34 & 51,53 \\
2 & Sapotaceae & 13,80 & 11,46 & 19,97 & 45,23 \\
3 & Chrysobalanaceae & 6,61 & 9,09 & 6,92 & 22,63 \\
4 & Myristicaceae & 7,60 & 5,53 & 5,21 & 18,34 \\
5 & Lauraceae & 5,63 & 4,34 & 7,99 & 17,96 \\
6 & Moraceae & 5,35 & 5,92 & 6,03 & 17,30 \\
7 & Caesalpiniaceae & 3,94 & 6,32 & 4,14 & 14,40 \\
8 & Burseraceae & 5,63 & 5,92 & 2,79 & 14,34 \\
9 & Mimosaceae & 4,22 & 5,53 & 4,50 & 14,25 \\
& & $H e c t a r e 2$ & & & \\
10 & Euphorbiaceae & 5,21 & 5,13 & 2,93 & 13,27 \\
1 & Lecythidaceae & 20,93 & 7,14 & 24,12 & 52,19 \\
2 & Sapotaceae & 11,44 & 11,18 & 14,18 & 36,80 \\
3 & Chrysobalanaceae & 10,14 & 10,24 & 8,10 & 28,48 \\
4 & Caesalpiniaceae & 7,15 & 6,21 & 6,47 & 19,83 \\
5 & Mimosaceae & 2,86 & 5,27 & 9,64 & 17,77 \\
6 & Euphorbiaceae & 6,89 & 6,21 & 4,68 & 17,78 \\
7 & Moraceae & 4,55 & 4,96 & 3,87 & 13,38 \\
8 & Myristicaceae & 4,29 & 4,96 & 3,64 & 12,89 \\
9 & Lauraceae & 2,73 & 3,10 & 1,96 & 7,79 \\
& & 1,95 & 1,24 & 3,89 & 7,08 \\
10 & Bombacaceae & 24,40 & 7,43 & 19,30 & 51,13 \\
1 & Lecythidaceae & 12,86 & 11,52 & 14,30 & 38,68 \\
2 & Sapotaceae & 5,66 & 7,06 & 6,85 & 22,57 \\
3 & Chrysobalanaceae & 5,77 & 4,83 & 7,17 & 17,77 \\
4 & Moraceae & 5,90 & 6,31 & 3,94 & 16,15 \\
5 & Myristicaceae & 5,24 & 8,55 & 2,28 & 16,07 \\
6 & Burseraceae & 4,59 & 7,06 & 4,30 & 15,95 \\
7 & Mimosaceae & 4,06 & 5,20 & 4,87 & 14,13 \\
8 & Caesalpiniaceae & 0,91 & 1,48 & 8,18 & 10,57 \\
9 & Combretaceae & 2,23 & 3,22 & 10,04 \\
\hline 10 & Arecaceae & 4,59 & & &
\end{tabular}

Den. $(\%)=$ densidade relativa; Div. $(\%)=$ diversidade relativa; Dom. $(\%)=$ dominância relativa IVIF = Indice de Valor de Importância das Familias 
médias de $51,62 \%$ e $40,24 \%$.

\section{Bibliografia citada}

Brasil. 1978. Levantamentos de Recursos Naturais. Ministerio das Minas e Energia (MME). Departamento Nacional da Produção Mineral (DNPM). Rio de Janeiro. Projeto Radambrasil.. Folha SB20, Purus, Vol. 17, 573p.

Cain, S.A.; Oliveira, G.M. de; Pires, J.M.; Silva, N.T. da . 1956. Aplication of some phytosociological tecniques to Brazilian rain forest. American Journal of Botany, 43(10):911-941.

Cain, S.A.; Castro, G.M.O. 1959. Manual of vegetation analysis. Hafner Publishing Company, New York, USA. 325 p.

Conceição, M. C. A. 1990. Análise estrutural de uma floresta de várzea no estado do Pará. Dissertação de Mestrado, UFPR. Curitiba, Paraná. $170 \mathrm{p}$.

Cronquist, A. 1981. An Integrated System of Classification of Flowers Plants. Columbia Univ. Press, New York, USA. 1261 p.
Curtis, J.T.; Mclntosch, R.P. 1950. The interrelation of certain analytic and synthetic phytosociological characters. Ecology, 31: 434-455.

Finol, U.H. 1971. Nuevos parametros a considerarse en el análisis estructural de las selvas virgines tropicales. Rev, For: Venez., 14(21):29-42.

Förster, M. 1973. Strukturanalyses eines tropischen Regenwaldes in Kolumbien. Allg. Forst. v. J. ZTG., 19(26):17-44.

Hosokawa, R. T. 1981. Manejo de florestas tropicais úmidas em regime de rendimento sustentado. Relatório técnico, UFPR. Curitiba, Paraná. 125 p.

Jardim, F.C. da S. 1985. Estrutura de floresta equatorial úmida da Estação Experimental de Silvicultura Tropical do INPA. Dissertação de Mestrado, INPA/FUA. Manaus, AM. 195 p.

Labouriau, L. F. G.; Matos Filho, A. 1948. Notas preliminares sobre a "região da Araucária" Anu. Bras. Econ. Flores., 1(1):215-228.

Lamprecht, H. 1962. Ensayo sobre unos

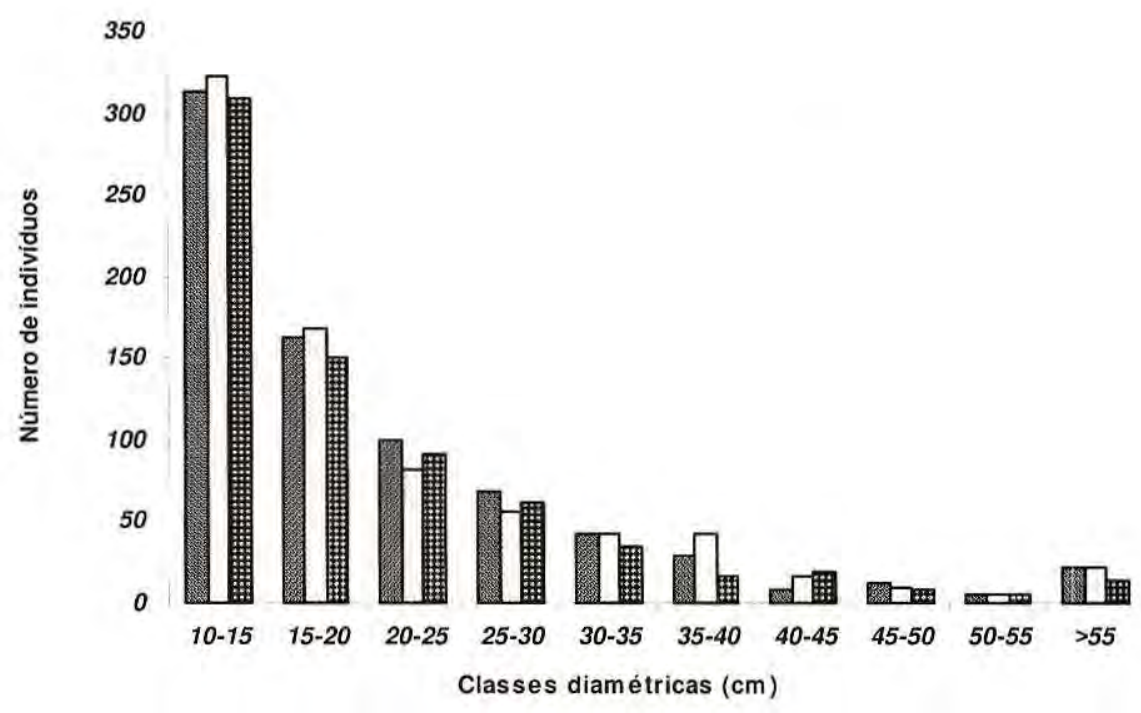

\section{Est.Fluídos $\square$ RUC-25 四 Aeroporto}

Figura 2. Frequência dos individuos por classe diamétrica, nos 3 ha na área do Rio Urucu-AM 
metodos para el analisis estructural de los bosques tropicales. Acta Cientifica Venezolana, 13(2):57-65.

Lamprecht, H. 1964. Ensayo sobre la Estructura floristica del parte sur-oriental del bosque universitário "El Caimital", Estado Baridas, Rev. For: Venez., 7(1011):77-119.

Lima Filho, D. A. 1996. Caracterização Floristica de 3 hectares de floresta de terra firme do Rio Urucu-AM. Dissertação de Mestrado, INPA/FUA. Manaus, Amazonas.162 p.

Longhi, S. V. 1980. A estrutura de uma floresta natural de Araucaria angustifolia (Bert.) O. Ktze, sul do Brasil. Dissertação de Mestrado, UFPR. Curitiba, Paraná.198 p.

Matos, F.D.A. \& Amaral, I.L. 1999. Análise ecológica de um hectare em floresta ombrófila densa de terra-firme, estrada da Várzea, Amazonas, Brasil. Acta Amazonica, 29(3):365-379.

Mori, A. S.; Boom, B. N.; Carvalho, A. M.; Santos, T. S, 1983. Ecological importance of Myrtaceae in an Eastern Brazilian wet forest (notes). Biotropica, 15(1):68-7.

PETROBRÁS. 1989. Relatório de Impacto Ambiental - RIMA. Urucu, Amazonas. p. 74-77

Revilla, J,; Lleras, E.; Rodrigues, W. A. - 1981. Levantamento, identificação e cadastramento da flora de Tucurui. Relatório técnico, Convênio INPA/ELN. Manaus-AM. 266 p.

Vega, C.L. 1966. Observaciones ecológicas sobre los bosques de roble de la sierra Boyacá. Colombia. Turrialba, 16(3):286296.

Vega, C.L. 1968. La estructura y composicion de los bosques humedos tropicales del Carare. Colombia. Turrialba, 18(4):416436.
Veloso, H.P.; Rangel Filho, A.L.R.; Lima, J.C.A. 1991. Classificação da vegetação brasileira adaptada a um Sistema Universal. Fundação Instituto Brasileiro de Geografia e Estatística - IBGE, Rio de Janeiro, Brasil. 124p. 